\title{
Evaluación del estado de conservación de los mamíferos de caza: un modelo comparativo en comunidades de la Reserva Nacional Pacaya Samiria (Loreto, Peru)
}

\author{
Evaluation of the conservation status of the game mammals: a comparative \\ model in communities of the Pacaya Samira National Reserve (Loreto, Peru)
}

\author{
Rolando Aquino ${ }^{1}$ y Arsenio Calle ${ }^{2}$
}

Presentado: $21 / 07 / 2003$

Aceptado: $\quad 20 / 08 / 2003$

\section{Resumen}

El presente trabajo contiene información relacionada a la caza, la abundancia y el impacto de la caza de los mamíferos que habitan los bosques inundables aledaños a dos comunidades asentadas en el interior de la Reserva Nacional Pacaya Samiria. Está basado en registros de caza y censos por transectos. Los registros de caza muestran un promedio de cosecha anual de 190,3 mamíferos del área de San Miguel, equivalente a 2275,9 kg de carne de monte, y 104,5 mamíferos del área de Parinari, equivalente a $985,9 \mathrm{~kg}$ de carne de monte. Los primates fueron los más abundantes en ambas áreas de estudio; sin embargo, las densidades estimadas fueron mayores para el área de Parinari. Ninguna especie de ungulados fue observada en el área de San Miguel, mientras que para el área de Parinari la densidad del tapir (Tapirus terrestris Linnaeus) fue estimada en 0,2 individuos/ $\mathrm{km}^{2}$ y para el pecarí labiado (Tayassu pecari Link) en 6,6 individuos $/ \mathrm{km}^{2}$. Finalmente, el modelo de cosecha sugiere sobrecaza para el tapir, maquisapa cenizo (Ateles belzebuth E. Geoffroy), choro (Lagothrix lagotricha Humboldt) y mono aullador (Alouatta seniculus Linnaeus), cuyas cosechas en algunos casos alcanzaron el $100 \%$ de la producción. Se discuten los factores que podrían influir para la escasez y/o ausencia de algunas especies, particularmente ungulados y primates.

Palabras claves: Reserva Nacional Pacaya Samiria, estado de conservación, densidad de fauna silvestre, impacto de caza.

\begin{abstract}
This paper contains information about the hunting, abundance and the hunting impact of the mammals that inhabit the lowland forests of the area surrounding two communities in the interior of the Pacaya Samiria National Reserve. It is based on hunting records and transect censuses. Hunting records show an average annual harvest of 190,3 mammals in the San Miguel area, equivalent to $2,275,9 \mathrm{~kg}$ of bushmeat; and 104,5 mammals in the Parinari area, equivalent to $985,9 \mathrm{~kg}$ of bushmeat. Primates were abundant in both study areas; however, the estimated densities were much higher in the Parinari area. No ungulates species were observed in the San Miguel area, whilst in the Parinari area the density of tapir (Tapirus terrestris Linnaeus) was estimated at 0,2 individuals/ $\mathrm{km}^{2}$ and that of white lipped peccary (Tayassu pecari Link) at 6, 6 individuals $/ \mathrm{km}^{2}$. Finally, the harvest model suggests overhunting of the tapir, white bellied spider monkey (Ateles belzebuth E. Geoffroy), common wooly monkey (Lagothrix lagotricha Humboldt) and red howler monkey (Alouatta seniculus Linnaeus), for which harvest in some cases reached $100 \%$ of the production. We discuss the factors that should influence the scarcity and/or absence of some species, particularly ungulates and primates.
\end{abstract}

Key words: Pacaya Samiria National Reserve, conservation status, wildlife animal densities, hunting impact.

\footnotetext{
${ }^{1}$ Instituto de Ciencias Biológicas Antonio Raimondi, Universidad Nacional Mayor de San Marcos, Estación Experimental del IVITA-Iquitos, Telefax 51-65-265510,

${ }^{2}$ Programa Integral de Desarrollo y Conservación Pacaya Samiria

Rolando Aquino email: ivit@tvs.com.pe
} 


\section{Introducción}

Los bosques inundables de la Amazonia peruana constituyen la despensa para los habitantes ribereños por los beneficios que obtienen de la pesca, caza y extracción de productos maderables y no maderables. La caza es una de las principales actividades que realizan las comunidades ribereñas para proveerse de proteína animal para subsistencia y para obtener un ingreso económico afín de cubrir las necesidades más apremiantes de salud y vestimenta. Esta actividad se incrementa durante la estación de «creciente» (febrero mayo), coincidente con la escasez de peces (Bodmer et al., 1999). La presión de caza a menudo a derivado en una sobrecaza y consecuentemente en una drástica reducción en las poblaciones de los animales de caza, creando un serio desequilibrio en los bosques inundables caracterizado por su alta diversidad pero que no soportan una caza desmedida. La Reserva Nacional Pacaya Samiria es un ejemplo de este tipo de bosques, donde alrededor de 200 comunidades asentadas en el entorno e interior hacen uso de sus recursos naturales, entre estas se encuentran las comunidades de San Miguel y Parinari.

La caza de animales silvestres por las comunidades locales asentadas en el cinturón e interior de la reserva es una realidad que debe ser aceptada y manejada de manera adecuada (Puertas et al., 2000), en caso contrario existe el riesgo de una sobrecaza y consecuentemente la extinción local, particularmente de los animales catalogados como de vida larga, como el tapir (Tapirus terrestris), los felinos y los primates (Peres, 1990; Robinson y Bodmer, 1999). El adecuado manejo será posible si contamos con suficiente información biológica de los animales de caza e información socioeconómica de aquellos que usan estos recursos (Bodmer et al., 1999). En consecuencia, la actual política de manejo de la fauna silvestre requiere que esté sustentada con: información de la biología de las poblaciones de los animales de caza, conocimiento de los patrones de cosecha y con el monitoreo a largo plazo que se lleve a cabo en un área de caza conocida para detectar declives en la población. La disponibilidad de estas informaciones permitiría la aplicación de modelos para evaluar el impacto de la caza.

Los cazadores de San Miguel y Parinari realizan actividades de caza en los bosques aledaños a sus respectivas comunidades. Estos bosques de acuerdo al «Plan Maestro» de la reserva corresponden a los denominados de uso directo y de protección estricta, respectivamente (INRENA, 2000). En ambas comunidades, desde enero de 1998 a diciembre del 2000 se compilaron los registros de caza, y entre octubre y noviembre del 2000 se ejecutaron censos por transectos en los bosques aledaños de ambas comunidades y cuyo propósito fue el de predecir si la caza estaba dentro de lo sostenible mediante el uso del modelo de cosecha diseñado por Bodmer et al. (1994). El modelo requiere estimados de: a) la producción anual (crías producidas/ individuo/ año) derivado de la productividad reproductiva (crías producidas/ hembra/ año) y la densidad poblacional y b) la presión de caza (individuos cosechados/ $\mathrm{km}^{2} /$ año).

\section{Áreas de estudio}

Los bosques aledaños a San Miguel y Parinari corresponden al típico bosque de várzea conformado por distintos tipos de vegetación, incluyendo a las «restingas» (altas y bajas), los chavascales (zonas abiertas) y los pantanos de palmeras asociación de Mauritia flexuosa $\mathrm{L}$. f. más conocidos como aguajales (Malleux, 1975; Encarnación, 1985). Estos bosques en época de «creciente» (febrero mayo) se inundan, quedando solamente pequeños montículos a manera de islas donde los animales terrestres pasan el invierno, por lo tanto la caza es mucho más fácil. Las restingas en ambas áreas de estudio apenas son fajas que varían entre 0,2 a $1,0 \mathrm{~km}$ de amplitud y máximo $4,0 \mathrm{~km}$ de longitud. Los aguajales y chavascales son los más extensos, este último es casi inhabitable por mantener una vegetación baja y muy cerrada, prácticamente impenetrables para el humano. Los aguajales en 


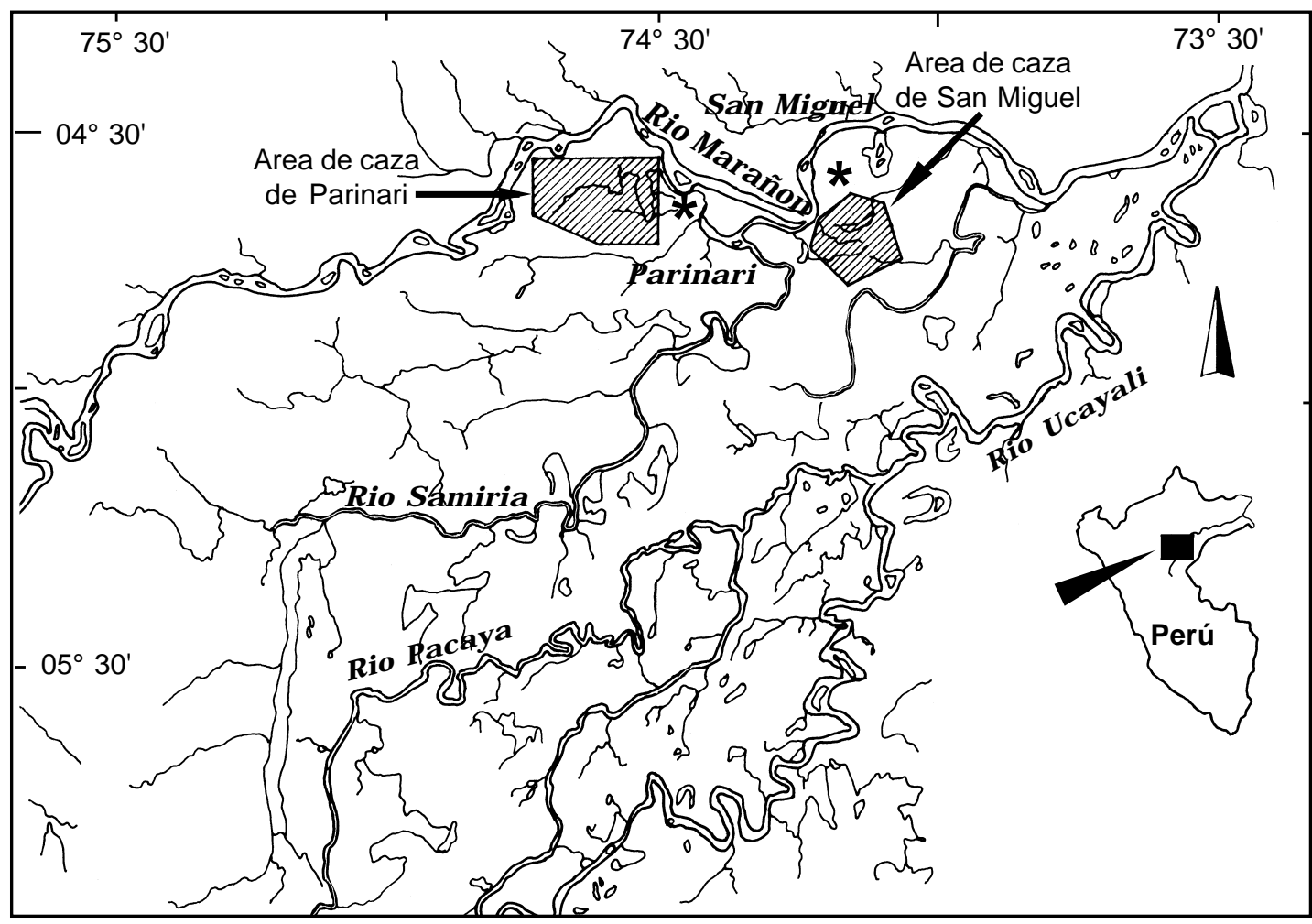

Figura 1. Mapa de la Reserva Nacional Pacaya Samiria mostrando las áreas de caza de San Miguel y Parinari.

ambas áreas de estudio predominan sobre las restingas. En los bosques de San Miguel están presentes los aguajales de origen reciente, cuyo piso permanece cubierto de agua durante todo el año, y los aguajales semieutrofizados cuyo piso se inunda temporalmente. En los bosques de Parinari predominan los aguajales semi-eutrofizados y eutrofizados, este último de piso casi compacto; ambos son visitados por mamíferos terrestres en época de vaciante.

\section{Material y Métodos}

Para evaluar el impacto de la caza fue necesario determinar la presión de caza (animales cazados $/ \mathrm{km}^{2}$ ). Para ello recurrimos a los registros de caza llevados a cabo de enero de 1998 a diciembre del 2000 en las localidades de San Miguel y Parinari por el Programa Integral de Desarrollo y Conservación Pacaya Samiria, AIF-WWF/DK. El área de caza fue estimada tomando como referencia los sitios donde fue- ron cazados los animales. El área de caza en San Miguel fue estimada en $100 \mathrm{~km}^{2}$ y en Parinari en $200 \mathrm{~km}^{2}$ (Fig. 1). Se agregó 30\% al promedio de animales cazados/ año para compensar los animales extraídos por otras comunidades vecinas a las áreas de estudio, cuya caza no fue registrada.

Para determinar el impacto de la caza para cada una de las áreas, además de la presión de caza, fue necesario estimar la densidad poblacional. Con este propósito, de octubre a noviembre del 2000 conducimos censos por transecto en cada una de las áreas de estudio. Los censos se realizaron en las restingas y aguajales donde habitualmente los moradores realizan sus actividades de caza; siendo excluidos los aguajales y restingas cercanos a los caseríos debido al diario trajín de los moradores para la cosecha de aguaje. También fueron excluidos los aguajales de origen reciente debido a su difícil acceso a pie. 
En San Miguel, tanto las restingas como los aguajales mostraron un aspecto muy disturbado, habiendo encontrado numerosas trochas en uso, así como abundantes campamentos. En los aguajales fue común encontrar los troncos derribados de aguaje y huasaí (Euterpe oleraceae Mart.) para la colecta de frutos y el palmito, respectivamente; por lo que advertimos cierta escasez de frutos de ambas especies. Los mamíferos terrestres fueron igualmente escasos y en algunos casos ciertas especies estuvieron ausentes como los ungulados.

En el área de Parinari, las restingas mostraron igualmente un aspecto alterado, habiendo encontrado trochas y campamentos en desuso. Los moradores de esta comunidad sostuvieron que los campamentos fueron construidos para fines de pesca y no para la caza. Los aguajales accesibles en época de vaciante no presentaron mayores signos de alteración que los troncos derribados de huasaí. En estos aguajales con abundante producción de frutos de aguaje, encontramos huellas relativamente abundantes de algunas especies de mamíferos terrestres, particularmente de algunos ungulados.

Para los censos se abrieron en los bosques de San Miguel nueve trochas con longitudes variables desde 3 a $5 \mathrm{~km}$ de longitud, cinco en restingas y cuatro en aguajales, mientras que en los bosques de Parinari se abrieron ocho trochas cuyas longitudes variaron entre 4 y $5,5 \mathrm{~km}$, cuatro en aguajales y cuatro en restingas. Por cada una de estas trochas se hicieron recorridos que fluctuaron desde 3 a 5 repeticiones. En San Miguel, fue cubierto $308 \mathrm{~km}$ de longitud censada y en Parinari 301 km (Tabla 1).
Para los censos fue utilizado el método de transecto lineal. Con esta finalidad, tres grupos conformado por dos observadores provistos de brújula, libreta de campo, lápiz y cinta métrica entre otros, recorrieron diariamente cada uno de los transectos a una velocidad promedio de $1,0 \mathrm{~km} /$ hora en restingas y de $0,5 \mathrm{~km} /$ hora en aguajales. Los censos fueron conducidos desde las 05:00 h a 11:00 h y de 14:00 a 18:00 h (hora local). Cada vez que hubo contacto con animales procedimos a anotar en la libreta de campo la especie, número de individuos (cuando se trataban de grupos ó manadas), tipo de bosque y la distancia perpendicular del primer animal avistado al transecto, este último permite estimar la densidad a partir de la línea de transecto de anchura fija usando la serie de expansión de Fourier (Brockelman y Ali, 1987; Buckland et al., 1993). Este método asume que todos los animales pueden ser observados dentro de una distancia predeterminada desde la línea con una probabilidad de $100 \%$. La línea fue la trocha usada para el censo. Para estimar la densidad solamente hemos considerado los avistamientos de animales dentro del ancho predeterminado para cada especie. Se anotó la longitud total censada y la densidad para los animales de caza más comunes fue estimada usando la siguiente fórmula: $\mathrm{D}=\mathrm{N} / 2 \mathrm{LW}$, donde $\mathrm{D}$ es la densidad, $\mathrm{N}$ es el número de animales avistados, $\mathrm{W}$ es el ancho predeterminado y L es la longitud de la trocha censada. De este modo $2 \mathrm{~W}$ es la amplitud censada. La desviación estándar (DS) fue calculada estimando las densidades independientemente para los diferentes transectos en línea y luego

Tabla 1. Apertura de trochas y longitudes recorridas en las áreas de estudio de San Miguel y Parinari.

\begin{tabular}{lccc}
\hline $\begin{array}{l}\text { Áreas de } \\
(\mathbf{k m})\end{array}$ & $\begin{array}{c}\text { \# de trochas } \\
\text { estudio }\end{array}$ & $\begin{array}{c}\text { Long. total } \\
\text { abiertas }\end{array}$ & $\begin{array}{c}\text { Long. Recorrida } \\
\text { de trochas } \mathbf{( k m )}\end{array}$ \\
\hline San Miguel & 9 & 36 & 308 \\
Parinari & 8 & 32 & 301 \\
Total: & 17 & 68 & 609 \\
\hline
\end{tabular}


se calculó un promedio. Para el caso de especies que viven en grupos y/o manadas, el número de individuos $/ \mathrm{km}^{2}$ fue calculado multiplicando el número de grupos por el tamaño promedio. El tamaño promedio fue estimado de aquellos grupos donde el conteo fue exacto. Para especies donde no fueron posible conteos exactos, el promedio corresponde a los obtenidos en otras áreas de la cuenca del río Samiria. Fueron excluidos de los censos los animales de hábitos nocturnos entre ellos los edentados, carnívoros $\mathrm{y}$ algunas especies de roedores.

El impacto de la caza para ungulados y primates en las áreas de estudio fue examinado usando el modelo de cosecha (Bodmer, 1994). Este modelo evalúa el impacto de la caza comparando la cosecha con la producción anual (número de crías/ individuo/ año) en un área de caza conocida. El modelo usa los estimados de: a) la productividad reproductiva (crías producidas/ hembra/ año) y b) ladensidad poblacional (Bodmer et al., 1997). La productividad reproductiva es determinada de los datos sobre la productividad bruta (número de fetos/ número de hembras adultas examinadas) y el número de gestaciones por año (Gottdenker, et al. 1997). Para el caso de ungulados, la producción anual resulta de multiplicar la productividad reproductiva por la mitad de la densidad poblacional (puesto que se asume que la mitad de la población son hembras). Los datos de cosecha y áreas de caza dieron un estimado de presión de caza (individuos cosechados $/ \mathrm{km}^{2}$ ).

Datos sobre tamaño de camada, número de gestaciones por año, proporción de hembras en actividad reproductiva tanto para ungulados como para primates con excepción del maquisapa cenizo, fueron tomados de Gottdenker et al. (1997) y Bodmer et al. (1999). Para el caso del maquisapa cenizo, el número de gestaciones/ año fue considerado del intervalo de nacimientos calculado para el maquisapa negro (Ateles paniscus Linnaeus) (McFarland, 1986: Symington, 1988). Por otro lado, a falta de datos sobre proporción de hembras adultas en actividad reproductiva en poblaciones naturales, ésta fue calculada de la proporción de infantes y juveniles encontradas en seis grupos de maquisapa cenizo en la cuenca del río Pucacuro, cuyas hembras adultas fueron identificadas y contadas (Aquino, obs. pers.). La densidad de hembras fue estimada considerando la proporción de 2:1 de hembras adultas sobre machos adultos en el maquisapa negro (McFarland 1986; Symington, 1988), de modo que asumimos que el $66 \%$ de la población del maquisapa cenizo está constituida por hembras.

Finalmente, a excepción del tapir, se asume que de los ungulados, un máximo del $40 \%$ de su producción puede ser cosechada sosteniblemente por tratarse de animales de vida corta (Robinson \& Redford, 1991). El tapir es un animal de vida larga y recomiendan que un máximo de $20 \%$ de su producción puede ser cosechada sosteniblemente. Los primates de tamaño grande son de vida larga y se asume que un máximo del $10 \%$ de su producción puede ser cosechada sosteniblemente. Justificaciones para este supuesto se encuentran en Robinson y Redford (1991) y Bodmer et al.(1997, 1999).

\section{Resultados}

\section{Mamíferos de caza en las áreas de estudio}

En los censos fue observado un total de 14 especies de mamíferos de caza en el área de San Miguel y 17 especies en el área de Parinari (Tabla 2). En ambas áreas, el mayor contacto ocurrió con primates y de éstos la mayoría a los denominados de tamaño mediano como el machín negro (Cebus apella Linnaeus), machín blanco (Cebus albifrons Humboldt) y huapo negro (Pithecia monachus E. Geoffroy) y de tamaño pequeño como el fraile (Saimiri boliviensis E. Geoffroy \& R. de Blainville) y pichico pardo (Saguinus fuscicollis Spix). Los bosques de San Miguel forman parte del área de distribución del maquisapa negro (Ateles paniscus Linnaeus); no obstante, durante los censos no hubo indicios de su presencia en esta parte de la reserva, lo cual indicaría su extinción local. En el área de Parinari no fue contactado ningún gru- 
po de tocón colorado (Callicebus cupreus Spix) y de pichico pardo, lo cual indicaría la ausencia de ambas especies en estos bosques. Los grupos observados, así como el tamaño promedio estimado para cada una de estas especies están contenidas en la Tabla 2.

\section{Presión de caza}

Los registros de caza nos indican un estimado anual de 190,3 mamíferos cazados del área de San Miguel 104,5 mamíferos del área de Parinari (Tabla 3).

En el área de San Miguel, los animales con mayor frecuencia de caza fueron los primates
(126,9 animales/ año) y ungulados (26,32 animales/año). Entre los primates, las especies más cazadas fueron el mono aullador, machín negro, machín blanco y el choro. Entre los ungulados, la especie más cazada fue el pecarí labiado con 22,5 animales/ año. En referencia a esta última especie, en los tres años de registro de caza en la comunidad de San Miguel (1998-2000) figuran la caza de 64 animales en 1998 y 6 en 1999, mientras que en el último año de registro prácticamente no hubo caza, lo que nos hace suponer que no hubo migración de esta especie hacia estos bosques. Es oportuno recalcar que durante los censos no hemos observado rastros

Tabla 2. Mamíferos de caza observados en las áreas de San Miguel y Parinari en el presente estudio. Para aquellos que viven en manadas ó grupos se utilizó el valor promedio, mientras que el resto se refiere a individuos contactados.

\begin{tabular}{|c|c|c|c|c|}
\hline \multirow[t]{2}{*}{ Mamiferos } & \multirow[t]{2}{*}{ Nombre común } & \multicolumn{2}{|c|}{$\begin{array}{c}\text { Grupos y/o indiv. } \\
\text { observados }\end{array}$} & \multirow[t]{2}{*}{$\begin{array}{c}\text { Tamaño } \\
\text { promedio }\end{array}$} \\
\hline & & S. Miguel & Parinari & \\
\hline \multicolumn{5}{|l|}{ Ungulados: } \\
\hline Tapirus terrestris & Tapir & 0 & 3 & \\
\hline Tayassu pecari & Pecarí labiado & 0 & 1 & 140,0 \\
\hline \multicolumn{5}{|l|}{ Primates: } \\
\hline Ateles belzebuth & Maquisapa cenizo & 0 & 6 & 7,3 \\
\hline Lagothrix lagotricha & Choro & 2 & 16 & 21,0 \\
\hline Alouatta seniculus & Mono aullador & 16 & 14 & 5,0 \\
\hline Cebus apella & Machín negro & 67 & 49 & 9,8 \\
\hline Cebus albifrons & Machín blanco & 19 & 34 & 17,0 \\
\hline Pithecia monachus & Huapo negro & 28 & 20 & 3,9 \\
\hline Callicebus cupreus & Tocón colorado & 7 & 0 & 2,7 \\
\hline Saimiri boliviensis & Fraile & 35 & 23 & 70,0 \\
\hline Saguinus fuscicollis & Pichico pardo & 48 & 0 & 5,8 \\
\hline \multicolumn{5}{|l|}{ Roedores: } \\
\hline Dasyprocta fuliginosa & Añuje & 1 & 1 & \\
\hline $\begin{array}{l}\text { Sciurus spp. } \\
\text { Carnivoros: }\end{array}$ & \multicolumn{3}{|c|}{ Carnívoros: } & \\
\hline Leopardus pardalis & Tigrillo & 0 & 1 & \\
\hline Nasuanasua & Achuni & 1 & 1 & 12,0 \\
\hline Eira barbara & Manco & 2 & 4 & \\
\hline Procyon cancrivorus & Perro conchero & 0 & 1 & \\
\hline \multicolumn{5}{|l|}{ Edentados: } \\
\hline Tamandua tetradactyla & Shihui & 1 & 1 & \\
\hline Bradypus variegatus & Pelejo & 2 & 1 & \\
\hline
\end{tabular}


de esta especie, este hecho indicaría su ausencia temporal o continua en esta parte de la reserva.

En el área de Parinari, los animales con mayor frecuencia de caza fueron los primates (75,6 animales/ año) y roedores (15,2 animales/ año). Entre los primates, el mono aullador y el machín blanco figuran entre los más ca- zados (Tabla 3). Entre los roedores, destaca por su mayor frecuencia de caza la paca ó majaz (Agouti paca Linnaeus).

En términos de biomasa, fueron extraídas un estimado de 2,275,9 kg de carne/ año del área de San Miguel y 985,9 kg/ año del área de Parinari. En San Miguel, las especies que contribuyeron con la mayor biomasa de carne

Tabla 3. Promedio anual estimado de extracción de mamíferos y de biomasa de las áreas de San Miguel y Parinari.

\begin{tabular}{|c|c|c|c|c|}
\hline \multirow[t]{2}{*}{ Especies } & \multicolumn{2}{|c|}{$\begin{array}{c}\text { Individuos extraídos } \\
\text { (Promedio anual) }\end{array}$} & \multicolumn{2}{|c|}{$\begin{array}{l}\text { Biomasa extraída (kg) } \\
\text { (Promedio anual) }\end{array}$} \\
\hline & S. Miguel & Parinari & S. Miguel & Parinari \\
\hline Ungulados: & 26,32 & 6,5 & $1,060,6$ & 374,3 \\
\hline Tayassu pecari & 22,75 & 3,9 & 750,7 & 128,7 \\
\hline Pecaritajacu & 1,3 & 0,65 & 32,5 & 16,2 \\
\hline Mazamaamericana & 0,65 & 0,65 & 21,4 & 21,4 \\
\hline Tapirus terrestris & 1,62 & 1,3 & 256,0 & 208,0 \\
\hline Primates: & 126,9 & 75,6 & 757,8 & 456,0 \\
\hline Ateles belzebuth & 0 & 1,3 & 0 & 14,3 \\
\hline Lagothrix lagotricha & 26,9 & 13,3 & 269,0 & 133,0 \\
\hline Alouatta seniculus & 35,1 & 24,0 & 280,8 & 192,0 \\
\hline Cebus apella & 33,1 & 14,6 & 115,8 & 51,1 \\
\hline Cebus albifrons & 28,6 & 20,8 & 85,8 & 62,4 \\
\hline Pithecia monachus & 3,2 & 1,6 & 6,4 & 3,2 \\
\hline Roedores: & 19,6 & 15,2 & 326,0 & 110,8 \\
\hline Agouti paca & 13,0 & 8,7 & 117,0 & 78,3 \\
\hline Dasyprocta fuliginosa & 0,6 & 6,5 & 3,5 & 32,5 \\
\hline Hydrochaeris hydrochaeris & 4,5 & 0 & 202,5 & 0 \\
\hline Sciurus sp. & 0,9 & 0 & 0,4 & 0 \\
\hline Coendu prehensilis & 0,6 & 0 & 2,6 & 0 \\
\hline Carnivoros: & 14,4 & $\mathbf{5 , 0}$ & 108,5 & 19,1 \\
\hline Nasuanasua & 2,2 & 2,2 & 6,6 & 6,6 \\
\hline Potos flavus & 6,5 & 1,6 & 13,0 & 3,2 \\
\hline Leopardus pardalis & 2,6 & 0,6 & 23,4 & 5,4 \\
\hline Panthera onca & 1,3 & 0 & 58,5 & 0 \\
\hline Herpailurus yagoaroundi & 0 & 0,3 & 0 & 3,0 \\
\hline Eira barbara & 0,9 & 0 & 4,3 & 0 \\
\hline Didelphys marsupialis & 0,9 & 0,3 & 2,7 & 0,9 \\
\hline Edentados: & $\mathbf{3 , 1}$ & 2,2 & $\mathbf{2 3 , 0}$ & 25,7 \\
\hline Myrmecophaga trydactyla & 0,3 & 0,6 & 9,0 & 18,0 \\
\hline Tamandua tetradactyla & 0,6 & 0 & 3,0 & 0 \\
\hline Bradypus variegatus & 0 & 0,3 & 0 & 1,2 \\
\hline Dasypus novemcinctus & 2,2 & 1,3 & 11,0 & 6,5 \\
\hline Total: & 190,32 & 104,5 & $2,275,9$ & 985,9 \\
\hline
\end{tabular}


fueron los ungulados y los primates. Entre los ungulados, la mayor biomasa correspondió al pecarí labiado con $750,7 \mathrm{~kg}$ / año y al tapir con $256 \mathrm{~kg} / \mathrm{año}$, respectivamente. Entre los primates, la mayor biomasa correspondió al mono aullador con $280,8 \mathrm{~kg} /$ año y al choro con $269 \mathrm{~kg} /$ año. En referencia al área de Parinari, las especies que contribuyeron con la mayor biomasa de carne fueron igualmente los primates y ungulados. Entre los primates la mayor biomasa correspondió al mono aullador con $192 \mathrm{~kg} /$ año y al choro con $133 \mathrm{~kg} /$ año. Entre los ungulados, la mayor biomasa correspondió al tapir con $208 \mathrm{~kg} /$ año y al pecarí labiado con $128 \mathrm{~kg}$ / año.

\section{Densidad y biomasa estimada}

Las densidades estimadas para cada una de las especies de hábito diurno para una y otra área de estudio están contenidas en la
Tabla 4. Los resultados indican claramente mayores densidades para especies de mayor biomasa para el área de Parinari y estuvieron representadas por el tapir, pecarí labiado, maquisapa cenizo y el choro. Estas especies probablemente ya no existen y/o son muy escasos en el área de San Miguel. Lo contrario ocurrió con las especies de tamaño pequeño, cuyas densidades fueron mayores para el área de San Miguel y menor para el área de Parinari y estuvieron representadas por el fraile y el pichico pardo. Al respecto, es posible que en ausencia de otros competidores por alimentos, las especies que quedan tienden a incrementar su población. Esto podría estar ocurriendo en el área de San Miguel con los machines, frailes y pichicos, cuyas densidades fueron las más altas en comparación a las demás especies de primates. Con excepción de los machines, las otras dos especies prácticamente no son caza-

Tabla 4. Densidad y biomasa estimada de mamíferos para las áreas de caza de San Miguel y Parinari.

\begin{tabular}{|c|c|c|c|c|c|}
\hline \multirow[t]{2}{*}{ Especies } & \multirow[t]{2}{*}{ Peso (kg) } & \multicolumn{2}{|c|}{ Densidad (ind. $/ \mathbf{k m}^{2}$ ) } & \multicolumn{2}{|c|}{ Biomasa $\left(\mathrm{Kg} / \mathbf{k m}^{2}\right)$} \\
\hline & & S. Miguel & Parinari & S. Migue & el Parinari \\
\hline \multicolumn{6}{|l|}{ Ungulados: } \\
\hline T. terrestris & 160 & 0 & 0,2 & 0 & 32 \\
\hline T. pecari & 33 & 0 & 6,6 & 0 & 217,8 \\
\hline \multicolumn{6}{|l|}{ Primates: } \\
\hline A. Belzebuth & 11 & 0 & 4,0 & 0 & 44,0 \\
\hline L. lagotricha & 10 & 4,4 & 37,2 & 44,0 & 372,0 \\
\hline A. seniculus & 8 & 6,2 & 5,5 & 49,6 & 44,0 \\
\hline C. apella & 3,5 & 66,6 & 50,0 & 233,1 & 175,0 \\
\hline C. albifrons & 3 & 30,2 & 56,6 & 90,6 & 169,8 \\
\hline P. monachus & 2 & 7,6 & 10,9 & 15,2 & 21,8 \\
\hline C. cupreus & 1,2 & 2,3 & 0 & 2,7 & 0 \\
\hline S. boliviensis & 0,8 & 248,7 & 167,2 & 199,0 & 133,7 \\
\hline S. fuscicollis & 0,5 & 56,8 & 0 & 26,4 & 0 \\
\hline \multicolumn{6}{|l|}{ Roedores: } \\
\hline D. fuliginosa & 5 & 0,1 & 0,5 & 0,1 & 0,5 \\
\hline Sciurus sp. & 0,4 & 13,2 & 25,0 & 5,3 & 10,0 \\
\hline \multicolumn{6}{|l|}{ Carnivoros: } \\
\hline L. pardalis & 9 & 0 & 0,1 & 0 & 0,9 \\
\hline N. nasua & 3 & 2,2 & 2,2 & 6,6 & 6,6 \\
\hline E. barbara & 4,8 & 0,8 & 1,6 & 3,8 & 7,6 \\
\hline Total: & & & & 678,3 & $1,235,7$ \\
\hline
\end{tabular}


Tabla 5. Modelo de cosecha de ungulados para las áreas de caza de San Miguel y Parinari.

\begin{tabular}{lrrrrr}
\hline & \multicolumn{2}{c}{ Pecari labiado } & & \multicolumn{2}{c}{ Tapir } \\
\cline { 2 - 3 } & S. Miguel & Parinari & S. Miguel Parinari \\
\cline { 2 - 3 } \cline { 5 - 6 } Productividad bruta & 0,51 & 0,51 & & 0,5 & 0,5 \\
\# gestaciones/ año & 1,6 & 1,6 & & 0,5 & 0,5 \\
Densidad media (indiv./km2) & 0,0 & 3,3 & & 0,0 & 0,1 \\
Producción anual (indiv./km2) & 0,0 & 2,69 & & 0,0 & 0,025 \\
Presión de caza (indiv./km2) & 0,22 & 0,02 & & 0,016 & 0,006 \\
\% producción cosechada & 100,00 & 0,7 & 100,0 & 24,0 \\
\hline
\end{tabular}

das para el consumo de subsistencia en ninguna de las áreas de estudio.

En referencia a la biomasa/ $\mathrm{km}^{2}$ estimada, ésta fue mayor para el área de Parinari (Tabla 4), lo cual es explicable por las densidades relativamente altas del pecarílabiado, choro, machín negro y machín blanco. En el área de San Miguel, la mayor biomasa/ $\mathrm{km}^{2}$ correspondió al machín negro, al machín blanco y al fraile.

\section{Impacto de la caza}

El impacto de la caza fue analizado únicamente para ungulados y primates de tamaño grande y mediano. Los roedores grandes como la paca y el añuje (Dasyprocta fuliginosa Wagler) no fueron analizados en este trabajo. La primera por no contar con información sobre densidad, por cuanto los censos se realizaron durante el día. No obstante, al añuje podemos considerarlo como especie sobrecazada en ambas áreas de estudio; al menos así reflejan los escasos registros de caza y el casi nulo contacto durante los censos. Las especies pequeñas como el pichico pardo, fraile y ardillas (Sciurus spp.), no han sido analizados porque éstos son los menos impactados por la caza o simplemente no son cazados, por lo tanto son los más abundantes en ambas áreas. Con las salvedades del caso, aquí presentamos el resultado del análisis del impacto de caza para el resto de las especies basado en el modelo de cosecha:

Pecarí labiado: El modelo de cosecha predice que esta especie está sujeto a una sobrecaza en el área de San Miguel. Se concluye esto porque el modelo indica una producción anual de 0,0 individuos $/ \mathrm{km}^{2}$ y los censos también nos indican que la población es 0,0 individuos. $/ \mathrm{km}^{2}$ por lo que la cosecha fue el $100 \%$. Lo contrario ocurrió en el área de Parinari, donde el modelo predice que la caza está dentro de lo sostenible con un $0,7 \%$ de su producción cosechada (Tabla 5).

Tapir: El modelo de cosecha indica que esta especie está siendo sobrecazada en ambas áreas de estudio. La extracción del 100\% de la producción en el área de San Miguel y del $24 \%$ en el área de Parinari, así lo confirman (Tabla 5).

Maquisapa cenizo: El modelo de cosecha indica que esta especie con distribución en la margen izquierda del río Samiria está siendo sobrecazada, por cuanto la cosecha alcanzó el 30\% de la producción, lo cual está muy por encima del 10\% sugerido por Bodmer et al.(1997, 1999) (Tabla 6).

Choro: El modelo de cosecha indica que esta especie está siendo sobrecazada en el área de estudio de San Miguel, donde los cazadores extrajeron el 100\% de la producción. En el área de Parinari, la cosecha probablemente está dentro de lo sostenible con un estimado de $3,7 \%$ de la producción cosechada (Tabla 6)

Mono aullador: El modelo de cosecha indica que esta especie en ambas áreas de estudio está siendo sobrecazada. En San Mi- 
Tabla 6. Modelo de cosecha de primates para las áreas de caza de San Miguel y Parinari.

\begin{tabular}{|c|c|c|c|c|c|}
\hline & \multicolumn{2}{|c|}{ Maquisapa cenizo } & \multicolumn{2}{|c|}{ Choro } & Mono aullador \\
\hline & Parinari & S. Miguel & Parinari & S. Miguel & Parinari \\
\hline Tamaño camada & 1,00 & 1,00 & 1,00 & 1,00 & 1,00 \\
\hline \# gestaciones/ año ${ }^{1,2}$ & 0,33 & 0,5 & 0,5 & 0,50 & 0,50 \\
\hline $\begin{array}{l}\text { Proporción hembras adulta } \\
\text { reproductiva activas }\end{array}$ & 0,25 & 0,30 & 0,30 & 0,25 & 0,25 \\
\hline Propor. hembras adultas 1,3 & 0,66 & 0,30 & 0,30 & 0,30 & 0,30 \\
\hline Densidad (ind./ $\mathbf{k m}^{2}$ ) & 4,00 & 4,4 & 37,2 & 6,20 & 5,50 \\
\hline Produc. Anual (ind./ km²) & 0,02 & 0,19 & 1,60 & 0,23 & 0,20 \\
\hline Presión de caza (ind./ $\mathbf{k m}^{2}$ ) & 0,006 & 0,27 & 0,06 & 0,35 & 0,12 \\
\hline \% producción cosechada & 30,00 & $>100,00$ & 3,70 & $>100,00$ & 60,00 \\
\hline
\end{tabular}

${ }^{1)}$ McFarland (1986); ${ }^{2)}$ Symington (1988); ${ }^{3)}$ White (1986).

guel, los niveles de cosecha alcanzaron el $100 \%$ de la producción y en Parinari el 60\% de la producción cosechada (Tabla 6).

Machín negro: El modelo de cosecha indica que la caza de esta especie en ambas áreas de estudio probablemente está dentro de lo sostenible, con un estimado de $5,1 \%$ y $1,4 \%$ de la producción cosechada, respectivamente.(Tabla 7).

Machín blanco: El modelo de cosecha indica que la cosecha de esta especie en ambas áreas de estudio probablemente está dentro de lo sostenible. Los estimados de cosecha alcanzaron el 7,2\% de la producción en San Miguel y el 1,3\% en Parinari (Tabla 7)
Huapo negro: El modelo de cosecha indica que esta especie probablemente está siendo cazada sosteniblemente en ambas áreas de estudio, por cuanto la cosecha no superó el $5,0 \%$ de la producción anual en ninguna de las áreas. (Tabla 7).

\section{Discusión}

La disminución de las poblaciones de la fauna silvestre está casi siempre relacionada con la sobrecaza, destrucción de su hábitat por deforestación y cosecha de frutos silvestres. En el caso específico del área de San Miguel, la caza y la cosecha de frutos (conjuntamente con la extracción del palmito de Euterpe

Tabla 7. Modelo de cosecha para primates de tamaño mediano en áreas de caza de San Miguel y Parinari.

\begin{tabular}{|c|c|c|c|c|c|c|}
\hline & \multicolumn{2}{|c|}{ Machin negro } & \multicolumn{2}{|c|}{ Machin blanco } & \multicolumn{2}{|c|}{ Huapo negro } \\
\hline & S.Miguel & Parinari & S.Miguel & Parinari & S.Migue & Parinari \\
\hline Tamaño camada ${ }^{1}$ & 1,00 & 1,00 & 1,00 & 1,00 & 1,00 & 1,00 \\
\hline$\#$ gestac./año ${ }^{1}$ & 0,57 & 0,57 & 0,57 & 0,57 & 0,48 & 0,48 \\
\hline $\begin{array}{l}\text { Proporción hembras adult. } \\
\text { reproduct. Activas } 1\end{array}$ & 0.57 & 0,57 & 0,80 & 0,80 & 0,50 & 0,50 \\
\hline Proporción hembras adult. ${ }^{1}$ & t. ${ }^{1} 0,30$ & 0,30 & 0,29 & 0,29 & 0,35 & 0,35 \\
\hline Densidad (ind./km²) & 66,6 & 50,00 & 30,2 & 56,6 & 7,6 & 10,90 \\
\hline Produc. Anual (ind. $/ \mathbf{k m}^{2}$ ) & 6,40 & 4,80 & 3,90 & 7,30 & 0,63 & 0,90 \\
\hline Presión caza (ind./km²) & 0,33 & 0,07 & 0,28 & 0,1 & 0,03 & 0,01 \\
\hline \% producción cosechada & 5,10 & 1,40 & 7,20 & 1,30 & 4,70 & 1,10 \\
\hline
\end{tabular}

\footnotetext{
1) McFarland (1986)
} 
oleraceae Mart.), podrían estar repercutiendo en las poblaciones de animales que habitan los diferentes tipos de bosques.

La mayor tasa de reproducción está en estrecha relación con la producción de frutos (Caughley, 1977). En los bosques inundables del Samiria existe poca diversidad de frutos en comparación a los bosques de altura; en consecuencia, son pocos los recursos alimenticios sobre los que se sustenta la alimentación de los ungulados, roedores, primates y aves. Los registros hasta ahora disponibles (Aquino, en prep.), indican que entre los principales recursos alimenticios de los animales de caza figuran los frutos y semillas de palmeras, así como de varias especies de moráceas y lecitidáceas. Los frutos del aguaje, huasaí (Euterpe oleracea Mart.), shapaja (Scheelea cephalotes Poepp. Ex Mart) y Pona (Iriartea exorrhiza Mart.), constituyen la base de la dieta de ungulados, roedores y primates (Bodmer, 1989; Bodmer et al., 1997; Allen, 1997). Desafortunadamente las dos primeras palmeras han sido exterminadas en los bosques aledaños a la comunidad de San $\mathrm{Mi}$ guel en un radio de más de $5 \mathrm{~km}$, afín de obtener los frutos y el palmito, lo cual podría ser un factor más para la disminución de las poblaciones de los animales de cazan. El ruido producido por la caída de los troncos de estas palmeras podría estar contribuyendo aún más a la fuga de animales entre los que se encuentran también algunas aves como los crácidos y tinámidos.

En el área de Parinari, la caza probablemente es el factor más importante para la disminución y/o desaparición de ciertas especies de mamíferos de importancia económica, entre los que podemos citar al tapir, pecarí de collar (Pecari tajacu Linnaeus), venado y los primates de tamaño grande como el maquisapa cenizo y el mono aullador. En las áreas donde se efectuaron los censos, los aguajales se mantuvieron casi intactos, salvo el huasaí que fue extraída casi en un 70\%. Gran parte del área de influencia de la comunidad de Parinari está conformado por aguajales y renacales (asociación de Ficus spp), siendo las restingas en una proporción quizá del $30 \%$. Esto nos indica que, por el momento, exceptuando las cercanías de la comunidad, estos bosques mantienen una alta productividad que garantizaría la supervivencia de las especies que habitan. En consecuencia, la escasa presencia y/o ausencia de ciertos ungulados, particularmente del tapir en el área de Parinari mas bien estaría relacionado con la alta presión de caza, la misma que habría derivado en una sobrecaza. Similares resultados fueron obtenidos para otras áreas de la Reserva Nacional Pacaya Samiria (Bodmer et al, 1997, 1999; Aquino et al., 2001) y otras cuencas del NorOriente peruano (Bodmer et al, 1994).

Finalmente, el modelo de cosecha no indica sobrecaza para los machines y huapo negro en ninguna de las dos áreas de caza. Estos resultados coinciden con los reportados por Bodmer et al.(1999) para el área de caza de Maipuco, Nueva Esperanza y San Antonio y por Aquino et al.(2001) para la cuenca del río Samiria, lo que nos hace presumir que por el momento son las únicas especies que estarían soportando la presión de caza en la Reserva Nacional Pacaya Samiria.

\section{Agradecimientos}

Dejamos constancia de nuestra gratitud al Programa Integral de Desarrollo y Conservación Pacaya Samiria, AIF-WWF/DK por facilitarnos los registros de caza. Nuestro reconocimiento a las autoridades políticas y moradores en general de San Miguel y Parinari por el apoyo incondicional durante nuestra estadía en ambas comunidades. Especial deferencia a Santos Apagueño en San Miguel y Juan Casternoque en Parinari, con quienes compartimos gratas experiencias durante las actividades de campo. A Pablo Puertas por la confianza depositada para la evaluación de la fauna en las comunidades citadas. Mi sincero reconocimiento a Ramón Noa, compañero e infatigable asistente de campo. 


\section{Literatura citada}

Allen, C. M. 1997. Frugivores, Palms and Conservation in Amazonia. M. Sc. Thesis, University of Florida, Gainesville.

Aquino, R.; R. E. Bodmer y J. G. Gil. 2001. Mamíferos de la cuenca del río Samiria: Ecología poblacional y sustentabilidad de la caza. Impr. Rosegraff. Lima, Perú.

Bodmer, R.E. 1989. Frugivory in Amazonian Artiodactyla: Evidence for the evolution of the ruminant stomach. J. Zoology 219: 457 - 467.

Bodmer, R. E. 1994. Managing wildlife with local communities in the Peruvian Amazon: The case of the Reserva Comunal Tamshiyacu-Tahuayo. In: D. Western y R. M. Wright (ed.): Natural connections: Perspectives in commnunitybased conservation. Island Press, Washington, D.C., 113 - 134 .

Bodmer, R.E.; P. Puertas, L. Moya y T. Fang. 1994. Estado de las poblaciones del tapir en la Amazonia peruana: En el camino de la extinción. Boletín de Lima 88: 33 - 42.

Bodmer, R. E.; R. Aquino y P. Puertas. 1997. Alternativas de manejo para la Reserva Nacional Pacaya Samiria: Un análisis sobre el uso sostenible de la caza. En: T. Fang; R. E. Bodmer; R. Aquino y M. Valqui (ed.): Manejo de Fauna Silvestre en la Amazonia. La Paz, Bolivia, 65 - 74.

Bodmer, R. E.; C. Allen, J. Penn, R. Aquino y C. Reyes. 1999. Evaluación del uso sostenible de la fauna silvestre en la Reserva Nacional Pacaya Samiria. Documento de Trabajo América Verde 4b. The Nature Conservancy, Arlington, USA.

Brockelman, R. y R. Alí. 1987. Methods of surveying and sampling forest primate population. In: C. W. Marash y R. A. Mittermeier (ed.) : Primate Conservation in Tropical Rain Forest. New York, 23 - 63.

Buckland, S.; K. Burham y J. Laake. 1993. Distance sampling: Estimating abundance of biological population. Chapman \& Hall, New York.

Caughley, G. 1977. Analysis of vertebrate populations. John Wiley and Songs, New York, N.Y.
Encarnación, F. 1985. Introducción a la flora y vegetación de la Amazonia Peruana: Estado actual de los estudios y un ensayo de una clave de determinación de las formaciones vegetales en la llanura amazónica. Candollea 40: 237 - 252.

Gottdenker, N.; R. E. Bodmer y P. Puertas. 1997. Ecología reproductiva de Tayassu pecari y de Tayassu tajacu en la Amazonia peruana. En: R. E. Bodmer; T. G. Fang; R. Aquino y M. Valqui (ed.): Manejo de Fauna Silvestre en la Amazonia. La Paz, Bolivia: 313 - 318.

INRENA, 2000. Plan Maestro de la Reserva Nacional Pacaya Samiria. Iquitos, Perú.

Malleux, O. J. 1975. Mapa Forestal del Perú (Memoria explicativa). Departamento de Manejo Forestal, Universidad Nacional Agraria, La Molina.

McFarland, M. 1986. Ecological determinants of fissionfusion sociality in Ateles and Pan. In: G. J. Else y P. C. Lee (ed.): Ecology and Conservation. Cambridge, Cambrigde University, $181-190$.

Peres, C.A. 1990. Effects on hunting on western Amazonian primates communities. Biological Conservation 54: $47-59$.

Robinson, J.G. y K. H. Redford. 1991. Sustainable harvest of neotropical forest mammal. In: J. G. Robinson y K. H. Redford (ed.): Neotropical Wildlife Use and Conservation. University of Chicago Press, Chicago, 415 - 429.

Robinson, J.G. y R.E. Bodmer. 1999. Towards wildlife management in tropical forests. J. of Wild. Manag. 63: $1-13$.

Symington, M. 1988. Demography, ranging patterns and activity budgets of black spider monkey (Ateles paniscus chameck) in the Manu National Park, Peru. Am. J. of Primatol. 15: 45 - 67.

Puertas, P.; R. Bodmer, J. López, J. Del Aguila y A. Calle. 2000. La importancia de la participación comunitaria en los planes de manejo de fauna silvestre en el nor-oriente peruano. Folia Amazónica (1-2): 159 - 179.

White, F. 1986. Census and preliminary observations on the ecology of black-faced black spider monkey (Ateles paniscus chamek) in Manu National Park. Am. J. of Primatol. 11: 125 - 132. 